\title{
Closed Gateways - Can Neuroprotectants Shield the Retina in Glaucoma?
}

\section{Thirumurthy Velpandian}

Department of Ocular Pharmacology and Pharmacy, Dr Rajendra Prasad Centre for Ophthalmic Sciences, All India Institute of Medical Sciences, New Delhi, India

\section{Abstract}

Neuroprotection as a pharmacological strategy to shield retinal ganglion cell death has been a popular approach adopted by many researchers. Yet, its effectiveness in preventing retinal ganglion cell death and thereby preserving vision in patients with primary open-angle glaucoma has not been demonstrated. ${ }^{[1]}$

Mitochondria are abundant in the unmyelinated part of the retinal ganglion cell axons to meet the higher energy demand for nerve conduction and for the maintenance of optimum neuronal function. ${ }^{[2]}$ Ischemia to the optic nerve head region can cause ganglion cell death at different rates. Therefore, effective intraocular pressure (IOP) control and blocking intracellular death triggers are both viable options. The right pharmacologic agent with a meaningful intraocular penetration would rationalize the neuroprotection strategy in glaucoma.

For the last three decades, several attempts have been made to establish a successful neuroprotectant for glaucoma using various in vitro and in vivo models. A comprehensive PubMed search with the MeSH terms "glaucoma" and "neuroprotection" revealed the following compounds, factors and plant extracts: NMDA receptor antagonists (memantine, dexanabinol), an $\alpha 2$-adrenergic receptor agonist (brimonidine), an $\alpha_{1}$-and $\beta$-adrenergic receptor antagonist with a nitric oxide donor (nipradilol), an angiotensin II type 1 receptor blocker (candesartan), a prostaglandin-related compound (tafluprost), calcium channel blockers (nilvadipine and cilnidipine), a sodium channel blocker (phenytoin), a dual 
sodium and calcium channel blocker (NS-7), a choline precursor (citicoline), a tetracycline (minocycline), an anti-inflammatory agent (aspirin [acetylsalicylic acid]), an endothelin antagonist (sulfisoxazole), an inducible nitric oxide synthase inhibitor (aminoguanidine), $\alpha$-tocopherol, and vinpocetine.

Endogenous compounds or their analogs, such as (endo)cannabinoids, granulocyte-colony stimulating factor (G-CSF), hematopoietic cytokine (erythropoietin), nerve growth factor, natural amino acid (taurine), glial cell line-derived neurotrophic factor and estrogen analogs (ZYC1, ZYC-3), were also scrutinized.

Plant extracts and derived compounds, such as Ginkgo biloba extract, carotenoids (xanthophylls), epigallocatechin-gallate, cannabinoids, ferulic acid from Ligusticum wallichii, a muscarnic agonist (pilocarpine), a lignophenol derivative from bamboo lignin (Lig-8), aloe-emodin metabolites, Lycium barbarum, red wine-polyphenols (resveratrol), Erigeron breviscapus, flavonoids, and spider venom derived 2-amino-5-ureidopentanamide from Parawixia bistriata, were suggested as potential candidates and some of them were also evaluated using in vitro and in vivo experimental models for neuroprotection in glaucoma.

Although there is good laboratory evidence for neuroprotection in glaucoma by many agents, proof from randomized clinical trials is lacking. ${ }^{[3]}$ The reasons for the failure of any such clinical attempts are unclear as many of the results from these trials have not been published.

A potential neuroprotectant compound needs to effectively cross the blood retinal barrier (BRB) into the eye. Agents given systemically, such as antimicrobials, seldom reach effective concentrations in the uninflammed eye. ${ }^{[4]}$ For that reason, a neuroprotectant may require multiple doses at frequent intervals over a longer duration to achieve a significant concentration in the eye. In addition to the plasma kinetics of a compound, its vitreous disposition is also important in determining its therapeutic action. It is currently well recognized that the presence of drug transporters in the BRB are responsible for the efflux of the intravitreally injected drug and for resisting the influx of the drug into the eye when it is given systemically. The presence of Pglycoprotein efflux transporters, cationic/anionic transporters, and other transporters in the BRB that resist the entry of systemically given substrates is well documented. ${ }^{[5-7]}$ Hence, for any predictable outcome in retinal neuroprotection, the ocular pharmacokinetic profile of the drug needs to be explored before initiating a clinical trial.

Systemic treatment with memantine of experimental glaucoma in monkeys showed that it was safe and effective in reducing the functional loss of the retina, although it had no effect on IOP. This study concluded that peak plasma concentration $\left(\mathrm{C}_{\max }\right)$ of memantine reached $1 \mu \mathrm{mol} / \mathrm{L}$ at $4-15$ months after the initiation of an oral dose of $4 \mathrm{mg} / \mathrm{kg} /$ day and similar levels were also observed in the vitreous humour after 16 months. ${ }^{[8]}$ Surprisingly, memantine, which is approved for the treatment of Alzheimer's disease, has failed to show any significant improvement in glaucoma in subsequent clinical trials as a neuroprotectant. ${ }^{[9]}$

A single $20 \mathrm{mg}$ dose of memantine resulted in a $\mathrm{C}_{\max }$ of $0.14 \mu \mathrm{mol} / \mathrm{L}$ in healthy human volunteers, a time to $\mathrm{C}_{\max }$ of 6.9 hours and a half-life of 62 hours. $^{[10]}$ A human study conducted by Kornhuber and Quack ${ }^{[11]}$ reported that a $20 \mathrm{mg} /$ day oral dose of memantine over 14 days resulted in concentrations of $0.37 \mu \mathrm{mol} / \mathrm{L}$ in the plasma and $0.207 \mu \mathrm{mol} / \mathrm{L}$ in the cerebrospinal fluid (CSF) at 2-3 hours after the last maintenance dose, and a CSF to serum ratio of 0.55 was proposed. A target concentration of memantine $1 \mu \mathrm{mol} / \mathrm{L}$ is expected to prevent excitotoxicity in neurons. ${ }^{[12]}$ The failure of memantine in the clinical trial for neuroprotection in glaucoma might have been due to an inadequate concentration of memantine in the vitreous after the oral dose of $20 \mathrm{mg}$ /day.

The randomized, double-blind, clinical trial reported by Zhong et al. ${ }^{[13]}$ in this issue of Drugs in $R \& D$ concluded that the Chinese traditional herb Erigeron breviscapus (vant.) Hand. Mazz. (EBHM) showed a partial protective effect in the visual field of glaucoma patients with controlled IOP. Breviscapine is a commercially produced plant extract from the Chinese herb 
EBHM. Scutellarin is a flavone glucuronide (5,6,4'-trihydroxyflavone-7-O-glucoronide) and is reported to be the main active component of EBHM. ${ }^{[14]}$ The percentage of scutellarin varies according to the source of production. In experimental studies, it has been reported to have estrogenic and neuroprotective properties. ${ }^{[15]}$ Breviscapine has been reported to attenuate neuroapoptosis and regulate the protein expression related to apoptosis after transient focal cerebral ischemia at a dose of $50-100 \mathrm{mg} / \mathrm{kg}$ in rats. ${ }^{[16]}$ Neuroprotective effects of scutellarin on brain ischemic injury-induced apoptosis have been reported to be associated with inhibition of PARP-dependent mitochondrial dysfunction and subsequent translocation of the apoptosisinducing factor pathway. ${ }^{[17]}$

Rat brain drug distribution studies showed that $22.3 \%$ of orally and $29.18 \%$ of tail vein administered radiolabeled breviscapine reached the brain tissues when it was administered at a dose of $0.4 \mathrm{mg} / \mathrm{kg} .{ }^{[18]}$ Interestingly, an experimental study in rats also reported that protective effects of scutellarin on cardiovascular and cerebrovascular ischemia were better than those of the extract mixture (breviscapine). ${ }^{[19]}$ Another study reported that the relative bioavailabilities of orally administered scutellarin and scutellarein conjugates in rats were very low $(<11 \%) .^{[20]}$

In this issue of Drugs in $R \& D$, Zhong et al. ${ }^{[13]}$ could not attribute the observed activity to any one of the individual active constituents of EBHM. They have correlated the activity to $4^{\prime}$-hydroscutellarein, $4^{\prime}$-hydrobaicalein-7- $\beta$-D-plamyagin, 4'-hydroscutellarein-7- $\beta$-D-glycuron methyl ester, pyromeconic acid, and several kinds of flavones and flavonoids. Chen et al. ${ }^{[21]}$ reported that after a single oral administration of scutellarin $60 \mathrm{mg}$ to 20 healthy subjects, plasma concentrations of scutellarin and its major metabolite (isoscutellarin) were found to reach a mean $\mathrm{C}_{\max }$ of $<5.0$ and $87 \mathrm{ng} / \mathrm{mL}$, respectively. They have also indicated that after hydrolysis in the gastrointestinal system by bacterial enzymes, the aglycone of scutellarin (scutellarein) was detected in serum as glucuronic acid conjugates, which showed regioselectivity and species difference. Considering the limited oral absorption of its major constituent (scutellarin), EBHM needs rigorous analysis regarding the active constituent(s) that get absorbed into the systemic circulation and, in turn, reach the vitreous humor for the projected activity.

Before promoting EBHM as a neuroprotectant for glaucoma and commencing with long-term studies, it is essential to identify the active constituent(s) and to determine whether the compounds have the ability to cross the closed gateways of the BRB to reach an effective concentration to shield the retina.

\section{Acknowledgments}

The article is supported in part by the Intramural grant of All India Institute of Medical Sciences, New Delhi, India.

\section{References}

1. Sena DF, Ramchand K, Lindsley K. Neuroprotection for treatment of glaucoma in adults. Cochrane Database Syst Rev 2010; (2): CD006539

2. Osborne NN. Mitochondria: their role in ganglion cell death and survival in primary open angle glaucoma. Exp Eye Res 2010 Jun; 90 (6): 750-7

3. Weinreb RN. Glaucoma neuroprotection: what is it? Why is it needed? Can J Ophthalmol 2007; 42 (3): 396-8

4. Velpandian $T$. Intraocular penetration of antimicrobial agents in ophthalmic infections and drug delivery strategies. Expert Opin Drug Deliv 2009; 6 (3): 255-70

5. Mannermaa E, Vellonen KS, Urtti A. Drug transport in corneal epithelium and blood-retina barrier: emerging role of transporters in ocular pharmacokinetics. Adv Drug Deliv Rev 2006; 58 (11): 1136-63

6. Tomi M, Hosoya KI. The role of blood-ocular barrier transporters in retinal drug disposition: an overview. Expert Opin Drug Metab Toxicol. Epub 2010 May 3

7. Senthilkumari S, Velpandian T, Biswas NR, et al. Evaluation of the impact of P-glycoprotein (P-gp) drug efflux transporter blockade on the systemic and ocular disposition of P-gp substrate. J Ocul Pharmacol Ther 2008; 24 (3): $290-300$

8. Hare WA, WoldeMussie E, Lai RK, et al. Efficacy and safety of memantine treatment for reduction of changes associated with experimental glaucoma in monkey, I: functional measures. Invest Ophthalmol Vis Sci 2004; 45 (8): 2625-39

9. Thomas SJ, Grossberg GT. Memantine: a review of studies into its safety and efficacy in treating Alzheimer's disease and other dementias. Clin Interv Aging 2009; 4: 367-77

10. Liu MY, Meng SN, Wu HZ, et al. Pharmacokinetics of single-dose and multiple-dose memantine in healthy Chinese volunteers using an analytic method of liquid 
chromatography-tandem mass spectrometry. Clin Ther 2008; 30 (4): 641-53

11. Kornhuber J, Quack G. Cerebrospinal fluid and serum concentrations of the N-methyl-D-aspartate (NMDA) receptor antagonist memantine in man. Neurosci Lett 1995; 195 (2): 137-9

12. Lipton SA. Paradigm shift in neuroprotection by NMDA receptor blockade: memantine and beyond. Nat Rev Drug Discov 2006; 5 (2): 160-70

13. Zhong Y, Xiang M, Ye W, et al. The visual field protective effect of Erigeron Breviscapus (vant.) Hand. Mazz. extract on glaucoma with controlled intraocular pressure: a randomized and double-blind clinical trial. Drugs R D 2010; 10 (2): $75-82$

14. Liu Q, Shi Y, Wang Y, et al. Metabolism profile of scutellarin in urine following oral administration to rats by ultra performance liquid chromatography coupled to timeof-flight mass spectrometry. Talanta 2009; 80 (1): 84-91

15. Zhu JT, Choi RC, Li J, et al. Estrogenic and neuroprotective properties of scutellarin from Erigeron breviscapus: a drug against postmenopausal symptoms and Alzheimer's disease. Planta Med 2009; 75 (14): 1489-93

16. Yiming L, Wei H, Aihua L, et al. Neuroprotective effects of breviscapine against apoptosis induced by transient focal cerebral ischaemia in rats. J Pharm Pharmacol 2008; 60 (3): $349-55$
17. Zhang HF, Hu XM, Wang LX, et al. Protective effects of scutellarin against cerebral ischemia in rats: evidence for inhibition of the apoptosis-inducing factor pathway. Planta Med 2009; 75 (2): 121-6

18. Shi SL, Xu LY, Wu JJ, et al. Comparison of the distribution of breviscapine in the brain by different administration routes. Yao Xue Xue Bao 2009; 44 (5): 515-8

19. Lin LL, Liu AJ, Liu JG, et al. Protective effects of scutellarin and breviscapine on brain and heart ischemia in rats. J Cardiovasc Pharmacol 2007; 50 (3): 327-32

20. Huang JM, Weng WY, Huang XB, et al. Pharmacokinetics of scutellarin and its aglycone conjugated metabolites in rats. Eur J Drug Metab Pharmacokinet 2005 Jul-Sep; 30 (3): 165-70

21. Chen X, Cui L, Duan X, et al. Pharmacokinetics and metabolism of the flavonoid scutellarin in humans after a single oral administration. Drug Metab Dispos 2006; 34 (8): $1345-52$

Correspondence: Dr Thirumurthy Velpandian, Department of Ocular Pharmacology and Pharmacy, Dr Rajendra Prasad Centre for Ophthalmic Sciences, All India Institute of Medical Sciences, New Delhi 110029, India.

E-mail: tvelpandian@hotmail.com 